\title{
CONFLICT-FREE VERTEX CONNECTION NUMBER AT MOST 3 AND SIZE OF GRAPHS
}

\author{
Trung DuY DoAn ${ }^{1}$ \\ School of Applied Mathematics and Informatics \\ Hanoi University of Science and Technology \\ Hanoi, Vietnam \\ e-mail: trungdoanduy@gmail.com \\ AND \\ INGO SCHIERMEYER \\ Institut für Diskrete Mathematik und Algebra \\ Technische Universität Bergakademie Freiberg \\ 09596 Freiberg, Germany \\ e-mail: Ingo.Schiermeyer@tu-freiberg.de
}

\begin{abstract}
A path in a vertex-coloured graph is called conflict-free if there is a colour used on exactly one of its vertices. A vertex-coloured graph is said to be conflict-free vertex-connected if any two distinct vertices of the graph are connected by a conflict-free vertex-path. The conflict-free vertex-connection number, denoted by $v c f c(G)$, is the smallest number of colours needed in order to make $G$ conflict-free vertex-connected. Clearly, $v c f c(G) \geq 2$ for every connected graph on $n \geq 2$ vertices.

Our main result of this paper is the following. Let $G$ be a connected graph of order $n$. If $|E(G)| \geq\left(\begin{array}{c}n-6 \\ 2\end{array}\right)+7$, then $v c f c(G) \leq 3$. We also show that $v c f c(G) \leq k+3-t$ for every connected graph $G$ with $k$ cut-vertices and $t$ being the maximum number of cut-vertices belonging to a block of $G$.

Keywords: vertex-colouring, conflict-free vertex-connection number, size of graph.
\end{abstract}

2010 Mathematics Subject Classification: 05C15, 05C40, $05 \mathrm{C} 07$.

\section{REFERENCES}

\footnotetext{
${ }^{1}$ This research is funded by the Hanoi University of Science and Technology (HUST) under project number T2018-PC-120.
} 
[1] S.A. van Aardt, C. Brause, A.P. Burger, M. Frick, A. Kemnitz and I. Schiermeyer, Proper connection and size of graphs, Discrete Math. 340 (2017) 2673-2677. doi:10.1016/j.disc.2016.09.021

[2] E. Andrews, E. Laforge, C. Lumduanhom and P. Zhang, On proper-path colorings in graphs, J. Combin. Math. Combin. Comput. 97 (2016) 189-207.

[3] V. Borozan, S. Fujita, A. Gerek, C. Magnant, Y. Manoussakis, L. Montero and Zs. Tuza, Proper connection of graphs, Discrete Math. 312 (2012) 2550-2560. doi:10.1016/j.disc.2011.09.003

[4] H. Chang, T.D. Doan, Z. Huang, S. Jendrol', X. Li and I. Schiermeyer, Graphs with conflict-free connection number two, Graphs Combin. 34 (2018) 1553-1563. doi:10.1007/s00373-018-1954-0

[5] H. Chang, Z. Huang, X. Li, Y. Mao and H. Zhao, Nordhaus-Gaddum-type theorem for conflict-free connection number of graphs. arXiv:1705.08316v1[math.CO].

[6] G. Chartrand, G.L. Johns, K.A. McKeon and P. Zhang, Rainbow connection in graphs, Math. Bohem. 133 (2008) 85-98.

[7] P. Cheilaris, B. Keszegh and D. Pálvölgyi, Unique-maximum and conflict-free coloring for hypergraphs and tree graphs, SIAM J. Discrete Math. 27 (2013) 1775-1787. doi:10.1137/120880471

[8] L. Chen, X. Li and Y. Shi, The complexity of determining the rainbow vertexconnection of a graph, Theoret. Comput. Sci. 412 (2011) 4531-4535. doi:10.1016/j.tcs.2011.04.032

[9] E. Chizmar, C. Magnant and P.S. Nowbandegani, Note on vertex and total proper connection numbers, AKCE Int. J. Graphs Comb. 13 (2016) 103-106. doi:10.1016/j.akcej.2016.06.003

[10] J. Czap, S. Jendrol' and J. Valiska, Conflict-free connections of graphs, Discuss. Math. Graph Theory 38 (2018) 911-920. doi:10.7151/dmgt.2036

[11] S. Jendrol', X. Li, Y. Mao, Y. Zhang, H. Zhao and X. Zhu, Conflict-free vertexconnections of graphs, Discuss. Math. Graph Theory 40 (2020) 51-65. doi:10.7151/dmgt.2116

[12] M. Ji, X. Li and X. Zhu, Conflict-free connections: algorithm and complexity, arXiv:1805.08072 (2018).

[13] H. Jiang, X. Li, Y. Zhang and Y. Zhao, On (strong) proper vertex-connection of graphs, Bull. Malays. Math. Sci. Soc. 41 (2018) 415-425. doi:10.1007/s40840-015-0271-5

[14] A. Kemnitz and I. Schiermeyer, Graphs with rainbow connection number two, Discuss. Math. Graph Theory 31 (2011) 313-320. doi:10.7151/dmgt.1547 
[15] M. Krivelevich and R. Yuster, The rainbow connection of a graph is (at most) reciprocal to its minimum degree, J. Graph Theory 63 (2010) 185-191.

doi:10.1002/jgt.20418

[16] X. Li and S. Liu, Tight upper bound of the rainbow vertex-connection number for 2-connected graphs, Discrete Appl. Math. 173 (2014) 62-69.

doi:10.1016/j.dam.2014.04.002

[17] X. Li and C. Magnant, Properly colored notions of connectivity - a dynamic survey, Theory Appl. Graphs 0(1) (2015) Art. 2. doi:10.20429/tag.2015.000102

[18] X. Li, Y. Mao and Y. Shi, The strong rainbow vertex-connection of graphs, Util. Math. 93 (2014) 213-223.

[19] X. Li and Y. Shi, On the rainbow vertex-connection, Discuss. Math. Graph Theory 33 (2013) 307-313. doi:10.7151/dmgt.1664

[20] X. Li, Y. Shi and Y. Sun, Rainbow connections of graphs: A survey, Graphs Combin. 29 (2013) 1-38. doi:10.1007/s00373-012-1243-2

[21] X. Li and Y. Sun, Rainbow Connections of Graphs ( Springer-Verlag, New York, 2012). doi:10.1007/978-1-4614-3119-0

[22] Z. Li and B. Wu, Maximum value of conflict-free vertex-connection number of graphs, Discrete Math. Algorithms Appl. 10 (2018) 1850059. doi:10.1142/S1793830918500593

[23] D.B. West, Introduction to Graph Theory (Prentice Hall, Upper Saddle River, 2001).

Received 18 September 2018 Revised 27 February 2019 Accepted 27 March 2019 